\title{
Dual Wavelet Frame Transforms on Manifolds and Graphs
}

\author{
Lihong Cui, ${ }^{1}$ Qiaoyun Wu, ${ }^{1}$ Jiale Liu, ${ }^{1}$ and Jianjun Sun $\mathbb{D}^{2}$ \\ ${ }^{1}$ Department of Mathematics, Beijing University of Chemical Technology, Beijing 100029, China \\ ${ }^{2}$ College of Chemical Engineering, Beijing University of Chemical Technology, Beijing, 100029, China
}

Correspondence should be addressed to Jianjun Sun; sunjj@mail.buct.edu.cn

Received 4 March 2019; Accepted 5 May 2019; Published 28 May 2019

Guest Editor: Mawardi Bahri

Copyright (C) 2019 Lihong Cui et al. This is an open access article distributed under the Creative Commons Attribution License, which permits unrestricted use, distribution, and reproduction in any medium, provided the original work is properly cited.

\begin{abstract}
In this paper, we consider the dual wavelet frames in both continuum setting, i.e., on manifolds, and discrete setting, i.e., on graphs. Firstly, we give sufficient conditions for the existence of dual wavelet frames on manifolds by their corresponding masks. Then, we present the formula of the decomposition and reconstruction for the dual wavelet frame transforms on graphs. Finally, we give a numerical example to illustrate the validity of the dual wavelet frame transformation applied to the graph data.
\end{abstract}

\section{Introduction}

Interest in signal processing algorithms in various applications has increased in recent years. Examples of these signal processing algorithms include sensor networks, transportation networks, the Internet, and social networks. In these applications, data are defined on topologically complicated domains, such as high-dimensional structures, irregularly sampled spaces, and manifolds. Such datasets are commonly referred to as big data. Several attempts have been made to extend conventional signal processing techniques to big data. As described in [1], the success of wavelet frames for data defined on flat domains has encouraged research on the generalization of wavelets and wavelet frames on topologically complicated domains. For example, Coifman and Maggioni constructed diffusion wavelets and diffusion polynomial frames on manifolds in [2,3]. Geller and Mayeli studied the construction of wavelets on compact differentiable manifolds in [4]. Hou et al. constructed the well-known Mexican hat wavelet on a manifold geometry in [5]. Graphs are effective ways to represent the geometric structures of data on complicated domains. Hammond et al. first used spectral graph theory to characterize spectral graph wavelets on graphs in [6]. Leonardi and Van De Ville introduced Meyer-like wavelets and scaling kernels that result in tight spectral graph wavelet frames in $[7,8]$. Shuman et al. first characterized a family of systems of uniformly translated kernels in the graph spectral domain that gave rise to tight frames of atoms generated via generalized translation on a graph in [9]. In addition, wavelet frames can be applied to sparse representation of piecewise smooth functions. Dong developed a system frame for sparse representation on graphs using tight wavelet frames and applications in [1]. Wang and Zhuang provided a complete characterization for the tightness of framelet systems in both continuous and discrete framelets on a manifold in [10]. Analogous to the standard Cohen-Daubechies-Feauveau (CDF) construction of factorizing a maximally flat Daubechies half-band filter, Narang and Ortega proposed the design of graph-QMF for arbitrary undirected weighted graphs in [11]. Tanaka and Sakiyama studied $M$-channel oversampled filter banks satisfying the perfect reconstruction condition for graph signals and showed oversampled graph filter banks for applications to graph signal denoising in [12].

As a generalization of tight wavelet frames, pairs of dual wavelet frames have proved particularly useful in signal denoising and many other applications where translation invariance or redundancy is important. Han first characterized homogeneous dual wavelet frames for any general dilation matrix in the space $F L_{2}(S)$ in [13]. In this case, $F L_{2}(S):=\left\{f \in L_{2}\left(\mathbb{R}^{n}\right) ; \operatorname{supp} \widehat{f} \subset S\right\}$, where $S$ denotes a measurable subset of $\mathbb{R}^{n}$. Chui et al. were the first to completely characterize tight dual frames with maximum vanishing moments generated using two generators derived from the same refinable function in [14]. Daubechies and Han constructed pairs of dual wavelet frames from two refinable 
functions in [15]. Ehler constructed compactly supported multivariate pairs of dual wavelet frames called biframes, based on the mixed oblique extension principle in [16]. Han and Shen generalized the mixed extension principle in $L_{2}\left(\mathbb{R}^{n}\right)$ to obtain and characterize dual Riesz bases in Sobolev spaces in [17]. Jiang and Pounds studied sixfold symmetric biframes with four framelets (frame generators) for triangular-mesh-based surface multiresolution processing in [18]. Readers can refer to [19-22] for more construction methods and properties regarding dual wavelet frameworks in $L_{2}\left(\mathbb{R}^{n}\right)$.

Motivated by these and other applications, in this paper, we introduce and study dual wavelet frames on manifolds. We consider the characteristics for the existence of dual wavelet frames in both continuous and discrete settings. In particular, discrete dual wavelet frame transforms and numerical examples on graphs are provided. The results may serve as an analysis tool for the processing of graph data. The paper is organized as follows. In Section 2, we briefly review some basic notations and concepts related to our present work. In Section 3, the conditions for the existence of dual wavelet frames on manifolds are proved. In Section 4, we discuss discrete dual wavelet frame transforms on graphs (DWFTG) and provide a multiresolution analysis (MRA) on a graph. For computation and applications in practice, in Section 5, we show that discrete dual wavelet frame transforms on graphs can be achieved by using low-degree Chebyshev polynomials. In addition, we give numerical simulations of fast dual wavelet frame transforms on graphs to demonstrate the efficient implementation of the framelet transforms.

The following is the list of notations used in this paper. Let $\mathbb{R}, \mathbb{N}$, and $\mathbb{Z}$ denote the set of real numbers and the set of natural number and integers, respectively. Let $z^{*}$ denote the conjugate of complex number $z$. Let $\ell_{2}(\mathbb{Z})$ be the set of 2 convergent sequences on $\mathbb{Z}$. Let $\mathscr{M}$ be a manifold. For a given compact, connected, and smooth Riemannian manifold $(\mathscr{M}, \mu)$, let $L_{2}(\mathscr{M})$ be a space of complex-valued square integrable functions on $\mathscr{M}$. Let $\Delta$ be the LaplaceBeltrami operator on $\mathscr{M}$ with respect to the metric $\mu . \delta_{\ell, \ell^{\prime}}$ is the Kronecker delta function with $\delta_{\ell, \ell^{\prime}}=1$ if $\ell=\ell^{\prime}$ and 0 otherwise. Moreover, let $G:=\{V, E, w\}$ denote a undirected, connected, weighted graphs, where $V:=\left\{v_{n} \in \mathscr{M}: n=\right.$ $1, \cdots, K\}$ is a discretization of a given manifold $\mathscr{M}, E \subset V \times V$ is an edge set, and $w: E \longmapsto \mathbb{R}^{+}$denotes a weight function. We consider here only finite graphs with $|V|=K<\infty$.

\section{Definitions and Concepts on Manifolds}

Throughout this paper, we will use the following notations for the inner product and norm for the space of $L_{2}(\mathscr{M})$.

$$
\begin{aligned}
\langle f, g\rangle & :=\int_{\mathscr{M}} f(x) g^{*}(x) \mathrm{d} \mu(x), \\
\|f\|_{L_{2}(\mathscr{M})} & :=\left(\int_{\mathscr{M}}|f(x)|^{2} \mathrm{~d} \mu(x)\right)^{1 / 2},
\end{aligned}
$$

where $f, g \in L_{2}(\mathscr{M}), \mathrm{d} \mu(\boldsymbol{x})$ (or simply $\mathrm{d} x$ ) denotes the measure from the area element of a Riemannian metric $\mu$ on
$\mathscr{M}$, a probability measure $\mu$ satisfies $\mu(\mathscr{M})=1$, and endowed with the $L_{2}$ norm.

Definition 1. Let $\Delta$ be the Laplace-Beltrami operator on $\mathscr{M}$, $\left\{u_{\ell}\right\}_{\ell=0}^{\infty} \subset L_{2}(\mathscr{M})$ and $\left\{\lambda_{\ell}\right\}_{\ell=0}^{\infty} \subset \mathbb{R}$; if the eigenvalue problem

$$
\Delta u_{\ell}+\lambda_{\ell}^{2} u_{\ell}=0
$$

holds, then the sequence $\left\{\left(u_{\ell}, \lambda_{\ell}\right)\right\}_{\ell=0}^{\infty}$ of pairs is called a welldefined eigensystem for $L_{2}(\mathscr{M})$.

Definition 2. The two sequences pairs $\left\{\left(u_{\ell}, \lambda_{\ell}\right)\right\}$ and $\left\{\left(\tilde{u}_{\ell}, \tilde{\lambda}_{\ell}\right)\right\}$ are said to be a biorthonormal eigenpair for $L_{2}(\mathscr{M})$, if they satisfy the following properties:

(1) A pair of sequences $u_{\ell}, \tilde{u}_{\ell} \subset L_{2}(\mathscr{M})$ form an biorthonormal basis of $L_{2}(\mathscr{M})$; i.e., $\left\langle u_{\ell}, \widetilde{u}_{\ell^{\prime}}\right\rangle=\delta_{\ell, \ell^{\prime}}$.

(2) Each of the two sequences $\left\{\lambda_{\ell}\right\}_{\ell=0}^{\infty},\left\{\widetilde{\lambda}_{\ell}\right\}_{\ell=0}^{\infty} \subset \mathbb{R}$ is a nondecreasing sequence of nonnegative numbers and satisfies $\lim _{\ell \rightarrow \infty} \lambda_{\ell}=\infty$ and $\lim _{\ell \rightarrow \infty} \widetilde{\lambda}_{\ell}=\infty$, respectively.

Since $\left\{\left(u_{\ell}, \lambda_{\ell}\right)\right\}$ and $\left\{\left(\widetilde{u}_{\ell}, \tilde{\lambda}_{\ell}\right)\right\}$ are an biorthonormal eigenpair for $L_{2}(\mathscr{M})$, the following can be given.

Definition 3. The generalized Fourier transform of a function $f \in L_{2}(\mathscr{M})$ is defined by

$$
\widehat{f}_{\ell}=\left\langle f, u_{\ell}\right\rangle_{L_{2}(\mathscr{M})}
$$

with $\widehat{f}_{\ell} \in \ell_{2}\left(\mathbb{Z}^{+}\right), \mathbb{Z}^{+}=\{0,1,2, \cdots\}$.

So, we can get the following result [6].

Theorem 4. Any $f \in L_{2}(\mathscr{M})$ has the Fourier expansion $f=$ $\sum_{\ell=0}^{\infty} \widehat{f}_{\ell} u_{\ell}$ in $L_{2}(\mathscr{M})$ and the Parseval relation

$$
\langle f, g\rangle=\sum_{\ell=0}^{\infty} \widehat{f}_{\ell} \widehat{g}_{\ell}^{*} .
$$

holds. In particular, when $f=g$, Parseval's identity

$$
\|f\|_{L_{2}(\mathscr{M})}^{2}=\sum_{\ell=0}^{\infty}\left|\hat{f}_{\ell}\right|^{2} .
$$

holds.

Let refinable function $\varphi=\psi_{0} \in L_{2}(R)$ and its dual function $\widetilde{\varphi}=\widetilde{\psi}_{0} \in L_{2}(R)$ which satisfy the following scaling equation:

$$
\begin{aligned}
& \widehat{\psi}_{0}(2 \xi)=\widehat{a}_{0}(\xi) \widehat{\psi}_{0}(\xi) \\
& \widehat{\widetilde{\psi}}_{0}(2 \xi)=\widehat{\widetilde{a}}_{0}(\xi) \widehat{\widetilde{\psi}}_{0}(\xi)
\end{aligned}
$$

\section{$\xi \in \mathbb{R}$}

where $\widehat{a}_{0}(\xi)=\sum_{k \in \mathbb{Z}} a_{0, k} e^{-i k \xi}, \widehat{\widetilde{a}}_{0}(\xi)=\sum_{k \in \mathbb{Z}} \widetilde{a}_{0, k} e^{-i k \xi}$ are all finitely supported and called a refinement mask or the low filter, respectively.

Given such a pair refinable function $\varphi=\psi_{0}$ and $\widetilde{\psi}_{0}$ with mask $\widehat{a}_{0}$ and $\widehat{\widetilde{a}}_{0}$, we define a pair functions set $\Psi$ := 
$\left\{\psi_{1}, \cdots, \psi_{r}\right\} \subset L_{2}(\mathbb{R})$ and $\widetilde{\Psi}:=\left\{\widetilde{\psi}_{1}, \cdots, \widetilde{\psi}_{r}\right\} \subset L_{2}(\mathbb{R})$ as follows:

$$
\begin{aligned}
& \widehat{\psi}_{j}(2 \xi)=\widehat{a}_{j}(\xi) \widehat{\psi}_{0}(\xi), \\
& \widehat{\widetilde{\psi}}_{j}(2 \xi)=\widehat{\widetilde{a}}_{j}(\xi) \widehat{\widetilde{\psi}}_{0}(\xi)
\end{aligned}
$$

$$
\xi \in \mathbb{R}
$$

where $\widehat{a}_{j}(\xi)=\sum_{k \in \mathbb{Z}} a_{j, k} e^{-i k \xi}$ and $\widehat{\tilde{a}}_{j}(\xi)=\sum_{k \in \mathbb{Z}} \widetilde{a}_{j, k} e^{-i k \xi}, j=$ $1,2, \cdots, r$ are called wavelet frame masks or the high pass filters of the system, respectively.

For $\psi_{j}$ and $\widetilde{\psi}_{j}$ defined by (7), denote $\Psi:=\left\{\psi_{1}, \cdots, \psi_{r}\right\} \subset$ $L_{2}(\mathbb{R})$ and $\widetilde{\Psi}=\left\{\widetilde{\psi}_{1}, \cdots, \widetilde{\psi}_{r}\right\} \subset L_{2}(\mathbb{R})$. So, there is the following definition of a quasi-affine system on manifold $\mathscr{M}$ [1].

Definition 5. Let $\Psi:=\left\{\psi_{1}, \cdots, \psi_{r}\right\} \subset L_{2}(\mathbb{R})$ be a set of functions. A quasi-affine system $X(\Psi) \subset L_{2}(\mathscr{M})$ is defined as

$$
X(\Psi):=\left\{\psi_{j, n, y}^{\mathscr{M}} \in L_{2}(\mathscr{M}): 1 \leq j \leq r, n \in \mathbb{Z}, y \in \mathscr{M}\right\},
$$

where

$$
\begin{aligned}
\psi_{j, n, y}^{\mathscr{M}}(x)=\sum_{\ell=0}^{\infty} \widehat{\psi}_{j}\left(2^{-n} \lambda_{\ell}\right) u_{\ell}^{*}(y) u_{\ell}(x), & \\
& n \in \mathbb{Z}, x \in \mathscr{M}, y \in \mathscr{M} .
\end{aligned}
$$

In fact, $\psi_{j, n, y}^{\mathscr{M}}(x)$ can be explained as the dilation and translation of $\psi_{j}$ at scale $n$ and a point $y \in \mathscr{M}$. And again, a similar definition for $X(\widetilde{\psi}) \subset L_{2}(\mathscr{M})$ can be given as follows:

$$
\begin{aligned}
& X(\widetilde{\Psi}) \\
& \quad:=\left\{\widetilde{\psi}_{j, n, y}^{\mathscr{M}} \in L_{2}(\mathscr{M}): 1 \leq j \leq r, n \in \mathbb{Z}, y \in \mathscr{M}\right\},
\end{aligned}
$$

where

$$
\begin{aligned}
& \tilde{\psi}_{j, n, y}^{\mathscr{M}}(x)=\sum_{\ell=0}^{\infty} \widehat{\widetilde{\psi}}_{j}\left(2^{-n} \tilde{\lambda}_{\ell}\right) \tilde{u}_{\ell}^{*}(y) \tilde{u}_{\ell}(x), \\
& \quad n \in \mathbb{Z}, x \in \mathscr{M}, y \in \mathscr{M} .
\end{aligned}
$$

Definition 6. Given a Hilbert space $\mathscr{H}$ and a set of vectors $\Gamma_{k} \in \mathscr{H}$, if there exist two positive constants $A$ and $B$ such that the inequality

$$
A\|f\|^{2} \leq \sum_{k}\left|\left\langle f, \Gamma_{k}\right\rangle\right|^{2} \leq B\|f\|^{2}
$$

holds for all $f \in \mathscr{H}$, then a set of vectors $\Gamma_{k} \in \mathscr{H}$ is called a frame in $\mathscr{H}$.

Now, we begin to give the definition of a pair of dual wavelet frame on manifolds.

Definition 7. For given $\Psi:=\left\{\psi_{1}, \cdots, \psi_{r}\right\} \subset L_{2}(\mathbb{R})$ and $\widetilde{\Psi}=$ $\left\{\tilde{\psi}_{1}, \cdots, \widetilde{\psi}_{r}\right\} \subset L_{2}(\mathbb{R})$, if
(1) $X(\Psi)$ and $X(\widetilde{\Psi})$ defined by (8) and (10) are in $L_{2}(\mathscr{M})(2)$ each of $X(\Psi)$ and $X(\widetilde{\Psi})$ is a wavelet frame in $L_{2}(\mathscr{M})(3)$ in $L_{2}$-sense, the following perfect reconstruction formula holds for any $f, g \in L_{2}(\mathscr{M})$

$$
f=\sum_{j=1}^{r} \sum_{n \in \mathbb{Z}} \int_{\mathscr{M}}\left\langle f, \psi_{j, n, y}^{\mathscr{M}}\right\rangle \widetilde{\psi}_{j, n, y}^{\mathscr{M}} \mathrm{d} y
$$

or, equivalently,

$$
\langle f, g\rangle_{L_{2}(\mathscr{M})}=\sum_{j=1}^{r} \sum_{n \in \mathbb{Z}} \int_{\mathscr{M}}\left\langle f, \psi_{j, n, y}^{\mathscr{M}}\right\rangle\left\langle\tilde{\psi}_{j, n, y}^{\mathscr{M}}, g\right\rangle \mathrm{d} y .
$$

Then the pair of systems $(X(\Psi), X(\widetilde{\Psi}))$ is called a dual wavelet frame for $L_{2}(\mathscr{M})$. In particular, when $\Psi=\widetilde{\Psi}$, the frame is said to be a tight frame for $L_{2}(\mathscr{M})$.

\section{Existences of Dual Wavelet Frames on Manifolds}

In this section, we give sufficient conditions for the existence of dual wavelet frames for $L_{2}(\mathscr{M})$ by their corresponding masks. First, we give Weyl's asymptotic formula $[23,24]$ and Griesers uniform bound of the eigenfunctions [25] as below similar to the way of literature $[1,10]$.

Lemma 8. Let $\left\{\left(u_{\ell}, \lambda_{\ell}\right)\right\}_{\ell=0}^{\infty}$ and $\left\{\left(\tilde{u}_{\ell}, \tilde{\lambda}_{\ell}\right)\right\}_{\ell=0}^{\infty}$ be well-defined eigensystem of the Laplace-Beltrami operator $\Delta$ on $M$; then

$$
\begin{aligned}
& \lambda_{\ell}=\ell^{1 / d}, \\
& \tilde{\lambda}_{\ell}=\ell^{1 / d} ;
\end{aligned}
$$

and

$$
\begin{gathered}
\left\|u_{\ell}\right\|_{L^{\infty}(\mathscr{M})} \leq c\left|\lambda_{\ell}\right|^{(d-1) / 2}, \\
\left\|\tilde{u}_{\ell}\right\|_{L^{\infty}(\mathscr{M})} \leq c\left|\tilde{\lambda}_{\ell}\right|^{(d-1) / 2} .
\end{gathered}
$$

where the constant $c$ depends only on the dimension $d \geq 2$ of the manifold. Here, the symbol $a_{n} \asymp b_{n}$ means that there exist positive constants $c, c^{\prime}$ independent of $n$ such that $0<c=$ $\liminf { }_{n}\left|a_{n} / b_{n}\right| \leq \lim \sup _{n}\left|a_{n} / b_{n}\right|=c^{\prime}<\infty$.

Lemma 9. Suppose a pair of functions $\left\{\psi_{j}: 0 \leq j \leq\right.$ $r\},\left\{\widetilde{\psi}_{j}, 0 \leq j \leq r\right\}$ in $L_{2}(\mathbb{R})$ and the corresponding pair of masks $\left\{\widehat{a}_{j}: 0 \leq j \leq r\right\},\left\{\widehat{\tilde{a}}_{j}, 0 \leq j \leq r\right\}$ satisfying equations (6) and (7). Let $\psi_{j, n, y}^{\mathscr{M}}$ and $\widetilde{\psi}_{j, n, y}^{\mathscr{M}}, j=0,1, \cdots, r$ be defined as in (9) and (11), respectively. If

$$
\begin{array}{r}
\left|\widehat{\psi}_{0}\right| \leq C_{0}(1+|\xi|)^{-s} \\
\text { and }\left|\widehat{\widetilde{\psi}}_{0}\right| \leq C_{0}(1+|\xi|)^{-s}
\end{array}
$$


then, for any $n \in \mathbb{Z}$,

$$
\begin{array}{r}
\sup _{y \in \mathscr{M}}\left\|\psi_{j, n, y}^{\mathscr{M}}\right\|_{L_{2}(\mathscr{M})}<\infty \\
\text { and } \sup _{y \in \mathscr{M}}\left\|\widetilde{\psi}_{j, n, y}^{\mathscr{M}}\right\|_{L_{2}(\mathscr{M})}<\infty .
\end{array}
$$

Similar to the literature [1], we can obtain the following results.

Theorem 10. Let $\left\{\psi_{j}: 0 \leq j \leq r\right\},\left\{\widetilde{\psi}_{j}: 0 \leq j \leq r\right\}$ in $L_{2}(\mathbb{R})$ be a pair functions with masks pair $\left\{\widehat{a}_{j}: 0 \leq j \leq r\right\},\left\{\widehat{\tilde{a}}_{j}\right.$ : $0 \leq j \leq r\}$ satisfying (6) and (7). Assume that the refinable function pair $\psi_{0}, \widetilde{\psi}_{0}$ satisfy conditions (17) and corresponding masks satisfy

$$
\begin{array}{r}
\left|\widehat{a}_{0}(\xi)-1\right| \leq C|\xi| \\
\text { and }\left|\widehat{\tilde{a}}_{0}(\xi)-1\right| \leq C|\xi|
\end{array}
$$

for $\xi$ near the origin. Then, the pair of systems $(X(\Psi), X(\widetilde{\Psi}))$ given by (8) and (10) forms a dual wavelet frame in $L_{2}(\mathscr{M})$ provided the equality

$$
\sum_{j=0}^{r} \widehat{a}_{j}^{*}(\xi) \widehat{\widetilde{a}}_{j}(\xi)=1
$$

holds for $\xi \in[-\pi, \pi]$.

Proof. For simplicity's sake, we define the operation $\mathscr{P}_{n, j}$ in $L_{2}(\mathscr{M})$ as

$$
\mathscr{P}_{n, j} f:=\int_{\mathscr{M}}\left\langle f, \psi_{j, n, y}^{\mathscr{M}}\right\rangle \widetilde{\psi}_{j, n, y}^{\mathscr{M}} \mathrm{d} y \text {, where } 0 \leq j \leq r .
$$

In particular,

$$
\mathscr{P}_{n} f:=\mathscr{P}_{n, 0} f:=\int_{\mathscr{M}}\left\langle f, \varphi_{n, y}^{\mathscr{M}}\right\rangle \widetilde{\varphi}_{n, y}^{\mathscr{M}} \mathrm{d} y .
$$

By formulas (9) and (11), we obtain

$$
\begin{aligned}
\widehat{\psi_{j, n, y}^{M}}[\ell] & =\widehat{\psi}_{j}\left(2^{-n} \lambda_{\ell}\right) u_{\ell}^{*}(y), \\
\text { and } \widehat{\widetilde{\psi}_{j, n, \cdot}^{M^{*}}}[\ell] & =\widetilde{\tilde{\psi}}_{j}^{*}\left(2^{-n} \widetilde{\lambda}_{\ell}\right) \tilde{u}_{\ell}^{*}(x),
\end{aligned}
$$

By Parseval's relation, we have

$$
\begin{aligned}
\left\langle f, \psi_{j, n, y}^{\mathscr{M}}\right\rangle & =\left\langle\widehat{f}, \widehat{\psi_{j, n, y}^{\mathscr{M}}}\right\rangle \\
& =\sum_{\ell=0}^{\infty} \hat{f}[\ell] \widehat{\psi}_{j}^{*}\left(2^{-n} \lambda_{\ell}\right) u_{\ell}(y) .
\end{aligned}
$$

Further,

$$
\left\langle\widehat{f, \psi_{j, n,}^{\mathscr{M}},}\right\rangle[\ell]=\widehat{f}[\ell] \widehat{\psi}_{j}^{*}\left(2^{-n} \lambda_{\ell}\right) .
$$

Therefore, we have

$$
\begin{aligned}
& \left(\mathscr{P}_{n, j} f\right)(x)=\left\langle\left\langle f, \psi_{j, n, n}^{\mathscr{M}}\right\rangle, \widetilde{\psi}_{j, n,}^{\mathscr{M}^{*}}\right\rangle \\
& =\left\langle\left\langle\widehat{f, \psi_{j, n,}^{M}}\right\rangle, \widehat{\widetilde{\psi}_{j, n, \cdot}^{\mathscr{M}}}\right\rangle \\
& =\sum_{\ell=0}^{\infty} \widehat{f}[\ell] \widehat{\psi}_{j}^{*}\left(2^{-n} \lambda_{\ell}\right) \widehat{\widetilde{\psi}}_{j}\left(2^{-n} \widetilde{\lambda}_{\ell}\right) \tilde{u}_{\ell}(x) .
\end{aligned}
$$

Thus,

$$
\begin{array}{r}
\widehat{\mathscr{P}_{n, j} f}[\ell]=\hat{f}[\ell] \widehat{\psi}_{j}^{*}\left(2^{-n} \lambda_{\ell}\right) \widehat{\widetilde{\psi}}_{j}\left(2^{-n} \widetilde{\lambda}_{\ell}\right) \\
\quad \text { for } 0 \leq j \leq r .
\end{array}
$$

By $\sum_{j=0}^{r} \widehat{a}_{j}^{*}(\xi) \hat{\tilde{a}}_{j}(\xi)=1$, we get

$$
\begin{aligned}
& \widehat{\mathscr{P}_{n, 0} f}[\ell]=\hat{f}[\ell] \widehat{\psi}_{0}^{*}\left(2^{-n} \lambda_{\ell}\right) \widehat{\widetilde{\psi}}_{0}\left(2^{-n} \widetilde{\lambda}_{\ell}\right)=\widehat{f}[\ell] \\
& \cdot \widehat{\psi}_{0}^{*}\left(2^{-n} \lambda_{\ell}\right) \widetilde{\widetilde{\psi}}_{0}\left(2^{-n} \lambda_{\ell}\right) \sum_{j=0}^{r} \widehat{a}_{j}^{*}\left(2^{-n} \lambda_{\ell}\right) \widehat{\tilde{a}}_{j}\left(2^{-n} \widetilde{\lambda}_{\ell}\right) \\
& =\sum_{j=0}^{r} \widehat{f}[\ell] \widehat{a}_{j}^{*}\left(2^{-n} \lambda_{\ell}\right) \widehat{\psi}_{0}^{*}\left(2^{-n} \lambda_{\ell}\right) \widehat{\tilde{a}}_{j}\left(2^{-n} \widetilde{\lambda}_{\ell}\right) \\
& \cdot \widetilde{\widetilde{\psi}}_{0}\left(2^{-n} \widetilde{\lambda}_{\ell}\right)=\sum_{j=0}^{r} \hat{f}[\ell] \widehat{\psi}_{j}^{*}\left(2^{-n+1} \lambda_{\ell}\right) \tilde{\widetilde{\psi}}_{j}\left(2^{-n+1} \widetilde{\lambda}_{\ell}\right) \\
& =\sum_{j=0}^{r} \widehat{\mathscr{P}}_{n-1, j} f[\ell] .
\end{aligned}
$$

Therefore,

$$
\mathscr{P}_{n} f=\sum_{j=0}^{r} \mathscr{P}_{n-1, j} f=\mathscr{P}_{n-1} f+\sum_{j=1}^{r} \mathscr{P}_{n-1, j} f,
$$

and hence

$$
\mathscr{P}_{n_{1}} f=\mathscr{P}_{n_{2}} f+\sum_{j=1}^{r} \sum_{n=n_{2}}^{n_{1}} \mathscr{P}_{n, j} f
$$

To obtain (13), we first need to prove that

$$
\lim _{n_{1} \longrightarrow+\infty} \mathscr{P}_{n_{1}} f=f, \quad f \in L_{2}(\mathscr{M}) .
$$

Due to

$$
\begin{aligned}
& \left\|\mathscr{P}_{n_{1}} f-f\right\|_{L_{2}(\mathscr{l})}^{2} \\
& \quad=\sum_{\ell=0}^{\infty}\left|\widehat{f}[\ell] \widehat{\varphi}^{*}\left(2^{-n_{1}} \lambda_{\ell}\right) \hat{\widetilde{\varphi}}\left(2^{-n_{1}} \widetilde{\lambda}_{\ell}\right)-\widehat{f}[\ell]\right|^{2} \\
& \quad=\sum_{\ell=0}^{\infty}|\widehat{f}[\ell]|^{2}\left|\widehat{\varphi}^{*}\left(2^{-n_{1}} \lambda_{\ell}\right) \hat{\widetilde{\varphi}}\left(2^{-n_{1}} \widetilde{\lambda}_{\ell}\right)-1\right|^{2} .
\end{aligned}
$$


By assumption (19), we have $\lim _{\xi \rightarrow 0} \widehat{\varphi}(\xi)=1, \lim _{\xi \rightarrow 0} \widehat{\widetilde{\varphi}}(\xi)=$ 1 ; thus, for each $\ell \geq 0$,

$$
\begin{aligned}
\lim _{n_{1} \longrightarrow+\infty} \widehat{\varphi}^{*}\left(2^{-n_{1}} \lambda_{\ell}\right) & =1 \\
\text { and } \lim _{n_{1} \longrightarrow+\infty} \widehat{\widetilde{\varphi}}\left(2^{-n_{1}} \tilde{\lambda}_{\ell}\right) & =1,
\end{aligned}
$$

and hence $\widehat{\varphi}$ and $\widehat{\widetilde{\varphi}}$ are bounded. Therefore, $\lim _{n_{1} \longrightarrow+\infty} \mathscr{P}_{n_{1}}\left\|\mathscr{P}_{n_{1}} f-f\right\|_{L_{2}(\mathscr{M})}^{2}=0$, and then

$$
\lim _{n_{1} \longrightarrow+\infty} \mathscr{P}_{n_{1}} f=f \text {. }
$$

Second, we show that

$$
\lim _{n_{2} \longrightarrow-\infty} \mathscr{P}_{n_{2}} f=0 \text {. }
$$

Since $\lim _{\xi \rightarrow \infty} \widehat{\varphi}(\xi)=0$ and $\lim _{\xi \rightarrow \infty} \widehat{\widetilde{\varphi}}(\xi)=0$, for $\ell \geq 0$, we have

$$
\begin{aligned}
& \lim _{n_{2} \longrightarrow-\infty} \widehat{\mathscr{P}_{n_{2}} f}[\ell] \\
& \quad=\lim _{n_{2} \longrightarrow-\infty} \widehat{f}[\ell] \widehat{\varphi}^{*}\left(2^{-n_{2}} \lambda_{\ell}\right) \widehat{\widetilde{\varphi}}\left(2^{-n_{2}} \widetilde{\lambda}_{\ell}\right)=0 .
\end{aligned}
$$

Therefore, by the boundedness of $\widehat{\varphi}$ and $\widehat{\widetilde{\varphi}}$, we have $\lim _{n_{2} \longrightarrow-\infty} \mathscr{P}_{n_{2}} f=0$.

This completes the proof of the theorem.

\section{Discrete Dual Wavelet Frame Transforms on Graphs}

Discrete dual wavelet frames on $\mathscr{M}$ are more desirable in practice. Graphs are usually understood as a certain discretization or a random sample from some smooth Riemannian manifold. In this section, we discuss discrete dual wavelet frame transforms on graphs.

For a given undirected, connected, weighted graphs $G:=$ $\{V, E, w\}$; let $A$ be a $K \times K$ adjacency matrix for a weighted graph $G$ with entries $\left(a_{m, n}\right)$, where

$$
\begin{aligned}
& a_{m, n} \\
& = \begin{cases}w\left(v_{m}, v_{n}\right), & \text { if } v_{m} \text { and } v_{n} \text { are connected by an edge in } E, \\
0, & \text { otherwise. }\end{cases}
\end{aligned}
$$

Let $D$ represent the degree matrix defined as follows:

$$
D=\operatorname{diag}\{d(1), d(2), \cdots, d(K)\},
$$

where $d(m)=\sum_{n} a_{m, n}$ is called the degree $d(m)$ of a vertex $v_{m}$.

The graph Laplacian plays an important role in the analysis and processing of graph data. The consistency of the graph Laplacian to the Laplace-Beltrami operator was studied in [26-28].

Definition 11. A nonnormalized graph Laplacian is defined as follows:

$$
\mathscr{L}=D-A .
$$

Let $\left\{\left(u_{k}, \lambda_{k}\right)\right\}_{k=0}^{K}$ be the set of pairs of eigenvalues and eigenfunctions of $\mathscr{L}$; then,

(1) without losing generality, we have $0=\lambda_{0} \leq \lambda_{1} \leq \lambda_{2} \leq$ $\cdots \lambda_{K-1}:=\lambda_{\text {max }}$

(2) the eigenfunctions form an orthonormal basis for all functions on the graph; that is, $\left\langle u_{k}, u_{k^{\prime}}\right\rangle=\delta_{k, k^{\prime}}$.

Definition 12. Let $f_{G}: V \longmapsto \mathbb{R}$ be a function on the graph $G$. Then its Fourier transform is defined by

$$
\widehat{f_{G}}[k]:=\left\langle f_{G}, u_{k}\right\rangle:=\sum_{n=1}^{K} f_{G}[n] u_{k}[n] \text {. }
$$

Suppose $f_{G}$ is sampled from the underlying function $f$ : $\mathscr{M} \longmapsto \mathbb{R}$ by $f_{G}\left(v_{k}\right):=\left\langle f, \varphi_{N, v_{k}}\right\rangle, v_{k} \in V$, where the dilation scale $N$ is the smallest integer such that $\lambda_{\text {max }}:=\lambda_{K-1} \leq 2^{N} \pi$. Therefore, its Fourier transform is given by

$$
\widehat{f_{G}}[k]=\widehat{f}_{k} \widehat{\varphi}^{*}\left(2^{-N} \lambda_{k}\right) \text {. }
$$

Note that the scale $N$ is selected such that $2^{-N} \lambda_{k} \in[0, \pi]$ for $0 \leq k \leq K-1$.

Given a graph function $f_{G}$, we define the discrete $L$-level dual wavelet frame decomposition operator as

$$
\begin{aligned}
\boldsymbol{W} f_{G}:= & \left\{W_{j, l} f_{G} ;(j, l) \in \mathbb{B}\right\}, \\
\mathbb{B}:= & \{(1,1),(2,1), \cdots,(r, 1),(1,2), \cdots,(r, L)\} \\
& \cup\{(0, L)\},
\end{aligned}
$$

where $W_{j, l} f_{G}$ is its dual wavelet frame coefficient. Let

$$
\boldsymbol{\alpha}=\boldsymbol{W} f_{G}=\left\{\alpha_{j, l}:(j, l) \in B\right\}, \quad \text { with } \alpha_{j, l}=W_{j, l} f_{G}
$$

and

$$
\begin{aligned}
& W^{T} \boldsymbol{\alpha}=\alpha_{0,0}, \\
& W_{j, l}^{T} \boldsymbol{\alpha}=\alpha_{0, l-1},
\end{aligned}
$$

where $\boldsymbol{W}^{T}$ is the reconstruction operator.

Now, we can define the discrete $L$-level dual wavelet frame decomposition and reconstruction algorithm for graph data as shown below.

Algorithm 13. Given a signal $f_{G}: V \longmapsto \mathbb{R}, f_{G}\left(v_{k}\right):=$ $\left\langle f, \varphi_{N, v_{k}}\right\rangle, v_{k} \in V$ and the associated trigonometric polynomials $\left\{\widehat{a}_{j}(\xi): 0 \leq j \leq r\right\}$ and $\left\{\widehat{\widetilde{a}}_{j}(\xi): 0 \leq j \leq r\right\}$, we assign $\widehat{\alpha}_{0,0}[k]=\widehat{f_{G}}[k]$. Then, the discrete $L$-level fast dual wavelet frame decomposition and reconstruction for $L_{2}(\mathscr{M})$ is given as follows (in the Fourier domain).

(1) Decomposition: for each $l=1,2, \cdots, L$.

(a) Obtain the low-frequency approximation of $\widehat{\alpha}_{0,0}[k]$ at level $l$ :

$$
\widehat{\alpha}_{0, l}[k]=\widehat{a}_{0}^{*}\left(2^{-N+l-1} \lambda_{k}\right) \widehat{\alpha}_{0, l-1}[k]
$$


(b) Obtain the dual framelet coefficients of $\widehat{\alpha}_{0,0}[k]$ at level $l$ :

$\widehat{\alpha}_{j, l}[k]=\widehat{a}_{j}^{*}\left(2^{-N+l-1} \lambda_{k}\right) \widehat{\alpha}_{0, l-1}[k], \quad j=1,2, \cdots, r$.

(2) Reconstruction: for each $j=L, L-1, \cdots, 1$,

$$
\widehat{\alpha}_{0, l-1}[k]=\sum_{j=0}^{r} \widehat{\widetilde{a}}_{j}\left(2^{-N+l-1} \lambda_{k}\right) \widehat{\alpha}_{j, l}[k] .
$$

Then we have the following perfect reconstruction formula from $\boldsymbol{\alpha}$ to $f_{G}$ through $\boldsymbol{W}^{T}$.

Theorem 14. If a given set of masks $\left\{a_{j}: 0 \leq j \leq r\right\}$ and $\left\{\widetilde{a}_{j}: 0 \leq j \leq r\right\}$ in $\ell_{1}(\mathbb{Z})$ satisfy (20), then, the discrete dual wavelet frame transforms $\boldsymbol{W}$ and $\boldsymbol{W}^{T}$ defined on $G=\{V, E, w\}$ satisfy

$$
\boldsymbol{W}^{T} \boldsymbol{W} f_{G}=f_{G} \quad \text { for all } f_{G}: V \longmapsto G .
$$

Proof. For simplicity, we only give proof in the case of $\mathrm{L}=2$ $L=2$. For a general $L$, it can be obtained in a similar way. For $L=2$, we have $\alpha:=\left\{\alpha_{j, l}:(j, l) \in \mathbb{B}\right\}$ with $\alpha_{j, l}:=W_{j, l} f_{G}$ and $\mathbb{B}=\{(j, l): 1 \leq j \leq r, l=1,2\} \cup\{(0,2)\}$. In accordance with (45) and (46), we have

$$
\widehat{\alpha}_{j, 2}[k]=\widehat{a}_{j}^{*}\left(2^{-N+1} \lambda_{k}\right) \widehat{a}_{0}^{*}\left(2^{-N} \lambda_{k}\right) \widehat{f_{G}}[k] .
$$

Therefore, by assuming $l=2$ in (47), we obtain

$$
\begin{aligned}
& \widehat{\alpha}_{0,2-1}[k]=\widehat{\alpha}_{0,1}[k]=\sum_{j=0}^{r} \widehat{\widetilde{a}}_{j}\left(2^{-N+1} \lambda_{k}\right) \widehat{\alpha}_{j, 2}[k] \\
& =\sum_{j=0}^{r} \widehat{\widetilde{a}}_{j}\left(2^{-N+1} \lambda_{k}\right) \widehat{a}_{j}^{*}\left(2^{-N+1} \lambda_{k}\right) \widehat{a}_{0}^{*}\left(2^{-N} \lambda_{k}\right) \widehat{f}_{G}[k] \\
& =\left(\sum_{j=0}^{r} \widehat{\widetilde{a}}_{j}\left(2^{-N+1} \lambda_{k}\right) \widehat{a}_{j}^{*}\left(2^{-N+1} \lambda_{k}\right)\right) \widehat{a}_{0}^{*}\left(2^{-N} \lambda_{k}\right) \\
& \cdot \widehat{f}_{G}[k]=\widehat{a}_{0}^{*}\left(2^{-N} \lambda_{k}\right) \widehat{f}_{G}[k] .
\end{aligned}
$$

By assuming $l=1$ in (47), we obtain

$$
\begin{aligned}
\widehat{\alpha}_{0,0}[k] & =\sum_{j=0}^{r} \widehat{\widetilde{a}}_{j}\left(2^{-N} \lambda_{k}\right) \widehat{\alpha}_{j, 1}[k] \\
& =\sum_{j=0}^{r} \widehat{\widetilde{a}}_{j}\left(2^{-N} \lambda_{k}\right) \widehat{a}_{j}^{*}\left(2^{-N} \lambda_{k}\right) \widehat{f}_{G}[k] \\
& =\left(\sum_{j=0}^{r} \widehat{\widetilde{a}}_{j}\left(2^{-N} \lambda_{k}\right) \widehat{a}_{j}^{*}\left(2^{-N} \lambda_{k}\right)\right) \widehat{f}_{G}[k] \\
& =\widehat{f_{G}}[k] .
\end{aligned}
$$

This shows that $\boldsymbol{W}^{T} \boldsymbol{W} f_{G}=f_{G}$.

\section{Polynomial Approximation and Numerical Simulations}

5.1. Polynomial Approximation and Fast DWFTG. Chebyshev polynomials have an important application in the approximation theory. In this section, we describe the details of the dual wavelet frame transform on graphs based on polynomial approximation by using the method proposed in literature $[1,6]$. One of the advantages of using lower Chebyshev polynomials to approach trigonometric polynomials is the efficiency of computing if the Laplacian is sparse.

We recall the relevant definitions and properties of the Chebyshev polynomials.

Definition 15. The Chebyshev polynomial $T_{k}(x)$ is defined as follows: $T_{k}(x)=\cos (\operatorname{karccos}(x)), x \in[-1,1]$.

Proposition 16. The Chebyshev polynomials have the following properties.

(1) $T_{k}(x)$ and $T_{j}(x)$ are orthogonal with respect to the weight function $1 / \sqrt{1-x^{2}}$ in the interval $[-1,1]$; that is,

$$
\begin{aligned}
\left\langle T_{k}, T_{j}\right\rangle & =\int_{-1}^{1} \frac{1}{\sqrt{1-x^{2}}} T_{k}(x) T_{j}(x) \mathrm{d} x \\
& =\int_{0}^{\pi} \cos k \theta \cos j \theta \mathrm{d} \theta \\
& = \begin{cases}\frac{\pi}{2} \delta_{k, j}, & k, j>0 ; \\
\pi, & k=j=0,\end{cases}
\end{aligned}
$$

where $x=\cos \theta$.

(2) $T_{k}(x)$ satisfies recursive relations

$$
\begin{aligned}
T_{0}(\xi) & =1, \\
T_{1}(\xi) & =\frac{\xi-\pi / 2}{\pi / 2}, \\
T_{k}(\xi) & =\frac{4}{\pi}\left(\xi-\frac{\pi}{2}\right) T_{k-1}(\xi)-T_{k-2}(\xi) .
\end{aligned}
$$

(3) For a given $g(\xi)$ with $\xi \in[0, \pi]$, it has a convergent Chebyshev series

$$
g=\frac{1}{2} c_{0}+\sum_{k=1}^{\infty} c_{k} T_{k}
$$

where the Chebyshev coefficients is $c_{k}=(2 / \pi) \int_{0}^{\pi} \cos (k \theta) g((\pi /$ $2)(\cos (\theta)+1) \mathrm{d} \theta$.

Thus, a smooth function $g(\xi)$ has the following accurate approximate:

$$
g(\xi) \approx \mathscr{T}^{n}(\xi)=\frac{1}{2} c_{0}+\sum_{k=1}^{n-1} c_{k} T_{k}
$$


and we denote the Chebyshev polynomial approximation of $\widehat{a}_{j}(\xi)$ and $\widehat{\tilde{a}}_{j}(\xi)$ as

$$
\begin{aligned}
\widehat{a}_{j}(\xi) & \approx \mathscr{T}_{j}^{n}(\xi)=\frac{1}{2} c_{j, 0}+\sum_{k=1}^{n-1} c_{j, k} T_{k}, \\
c_{j, k} & =\frac{2}{\pi} \int_{0}^{\pi} \cos (k \theta) \widehat{a}_{j}\left(\frac{\pi}{2}(\cos (\theta)+1)\right) \mathrm{d} \theta ; \\
\widehat{\tilde{a}}_{j}(\xi) & \approx \widetilde{\mathscr{T}}_{j}^{n}(\xi)=\frac{1}{2} \widetilde{c}_{j, 0}+\sum_{k=1}^{n-1} \widetilde{c}_{j, k} T_{k}, \\
\widetilde{c}_{j, k} & =\frac{2}{\pi} \int_{0}^{\pi} \cos (k \theta) \widetilde{\tilde{a}}_{j}\left(\frac{\pi}{2}(\cos (\theta)+1)\right) \mathrm{d} \theta .
\end{aligned}
$$

$$
\widetilde{U W_{j, l} f_{G}}= \begin{cases}U \hat{a}_{j}^{*}\left(2^{-N} \Lambda\right) U^{T} U \widehat{f_{G}}, & l=1, \\ U \widehat{a}_{j}^{*}\left(2^{-N+l-1} \Lambda\right) \widehat{a}_{0}^{*}\left(2^{-N+l-2} \Lambda\right) \cdots \widehat{a}_{0}^{*}\left(2^{-N} \Lambda\right) U^{T} U \widehat{f_{G}}, & 2 \leq l \leq L .\end{cases}
$$

However,

$$
\begin{gathered}
{\left[\begin{array}{cccc}
u_{0}[0] & u_{1}[0] & \cdots & u_{K-1}[0] \\
u_{0}[1] & u_{1}[1] & \cdots & u_{K-1}[1] \\
\vdots & \vdots & \ddots & \vdots \\
u_{0}[K-1] & u_{1}[K-1] & \cdots & u_{K-1}[K-1]
\end{array}\right]\left[\begin{array}{c}
\widehat{W_{j, l} f_{G}}[0] \\
\widehat{W_{j, l} f_{G}}[1] \\
\vdots \\
\widehat{W_{j, l} f_{G}}[K-1]
\end{array}\right]} \\
=\left[\begin{array}{c}
W_{j, l} f_{G}[0] \\
W_{j, l} f_{G}[1] \\
\vdots \\
W_{j, l} f_{G}[K-1]
\end{array}\right]
\end{gathered}
$$

where $W_{j, l} f_{G}[p]=\sum_{k=0}^{K-1} \widehat{W_{j, l} f_{G}}[k] u_{k}[p]$; i.e., $U \widehat{W_{j, l} f_{G}}=$ $W_{j, l} f_{G}$, and
The Laplacian $\mathscr{L}$ is a symmetric matrix so that the $\operatorname{diag}\left\{\lambda_{0}, \lambda_{1}, \cdots, \lambda_{K-1}\right\}$ and columns of $U$ are the eigenvectors. Now, we can use the above approximation to speed up the decomposition transform. First, we can rewrite the decomposition transform in the following matrix form:

$$
\begin{aligned}
& \widehat{W_{j, l} f_{G}} \\
& = \begin{cases}\widehat{a}_{j}^{*}\left(2^{-N} \Lambda\right) \widehat{f_{G}}, & l=1, \\
\widehat{a}_{j}^{*}\left(2^{-N+l-1} \Lambda\right) \widehat{a}_{0}^{*}\left(2^{-N+l-2} \Lambda\right) \cdots \widehat{a}_{0}^{*}\left(2^{-N} \Lambda\right) \widehat{f_{G}}, & 2 \leq l \leq L .\end{cases}
\end{aligned}
$$

where $\widehat{a}_{j}^{*}(\gamma \Lambda)=\operatorname{diag}\left\{\widehat{a}_{j}^{*}\left(\gamma \lambda_{0}\right), \hat{a}_{j}^{*}\left(\gamma \lambda_{1}\right), \cdots, \widehat{a}_{j}^{*}\left(\gamma \lambda_{K-1}\right)\right\}$.

Due to $U^{T} U=I$, we have eigenvalue decomposition $\mathscr{L}=U \Lambda U$ exists, where $\Lambda=$ 
Next, reconstruction transform $\boldsymbol{W}^{T}$ can be obtained similarly; i.e., (47) can be rewritten as the following matrix form:

$$
\left[\begin{array}{c}
\widehat{W_{0, l-1} f_{G}}[0] \\
\widehat{W_{0, l-1} f_{G}}[1] \\
\vdots \\
\widehat{W_{0, l-1} f_{G}}[K-1]
\end{array}\right]=\left[\begin{array}{cccc}
\sum_{j=0}^{r} \widehat{\tilde{a}}_{j}\left(2^{-N+l-1} \lambda_{0}\right) & 0 & \cdots & 0 \\
0 & \sum_{j=0}^{r} \widehat{\widetilde{a}}_{j}\left(2^{-N+l-1} \lambda_{1}\right) & \cdots & 0 \\
\vdots & \vdots & \ddots & \vdots \\
0 & 0 & \cdots & \sum_{j=0}^{r} \widehat{\tilde{a}}_{j}\left(2^{-N+l-1} \lambda_{K-1}\right)
\end{array}\right]\left[\begin{array}{c}
\widehat{W_{j, l} f_{G}}[0] \\
\widehat{W_{j, l} f_{G}}[1] \\
\vdots \\
\widehat{W_{0, l} f_{G}}[K-1]
\end{array}\right]
$$

Therefore, the above formula can be equivalently written in physical domain:

$$
W_{0, l-1} f_{G}=\sum_{j=0}^{r} U \widehat{\widetilde{a}}_{j}\left(2^{-N+l-1} \Lambda\right) U^{T} W_{j, l} f_{G}
$$

We can obtain the fast dual wavelet frame reconstruction transform on graphs as follows:

$$
W_{0, l-1}^{T} \boldsymbol{\alpha}=\sum_{j=0}^{r} \widetilde{\mathscr{T}}_{j}^{n}\left(2^{-N+l-1} \mathscr{L}\right) W_{j, l} f_{G} .
$$

And we have $\boldsymbol{W}^{T} \boldsymbol{W} \approx \boldsymbol{I}$.

5.2. Numerical Simulations of DWFTG. In this section, we give a numerical example to illustrate the validity of the dual wavelet frame transformation applied to the graph data.

Example 17 (linear). Let $\widehat{a}_{0}(\xi)=\widehat{\widetilde{a}}(\xi)=(1 / 4) e^{i \xi}\left(1+e^{-i \xi}\right)^{2}$ be the refinement masks of the continuous linear B-splines $\varphi$ and $\widetilde{\varphi}$ supported on $[-1,1]$. Define

$$
\begin{aligned}
& \widehat{a}_{1}(\xi)=-\frac{1}{2} e^{i \xi}\left(1-e^{-i \xi}\right)^{2}, \\
& \widehat{\widetilde{a}}_{1}(\xi)=\frac{1}{8} e^{i \xi}\left(1+e^{-i \xi}\right)^{2}, \\
& \widehat{a}_{2}(\xi)=-\frac{1}{2}\left(1-e^{-i \xi}\right)^{2}, \\
& \widehat{\widetilde{a}}_{2}(\xi)=\frac{1}{2} e^{-i \xi} .
\end{aligned}
$$

Then, this group of masks satisfy (20). Hence, the system pair $(X(\Psi), X(\widetilde{\Psi}))$ defined in (8) and (10) associated with the mask $\left\{\widehat{a}_{0}, \widehat{a}_{1}, \widehat{a}_{2}\right\},\left\{\widehat{\tilde{a}}_{0}, \widehat{\tilde{a}}_{1}, \widehat{\widetilde{a}}_{2}\right\}$ is a dual wavelet frame for $L_{2}(\mathscr{M})$.

Example 18 (cubic). Let $\widehat{a}_{0}(\xi)=(1 / 4) e^{i \xi}\left(1+e^{-i \xi}\right)^{2}$ be the refinement masks of the continuous linear B-splines $\varphi$ supported on $[-1,1]$ and $\widehat{\widetilde{a}}_{0}(\xi)=(1 / 16) e^{i 2 \xi}\left(1+e^{-i \xi}\right)^{4}$ be the refinement masks of the $C^{2}$ cubic B-splines $\widetilde{\varphi}$ supported on $[-2,2]$. Define

$$
\begin{aligned}
& \widehat{a}_{1}(\xi)=-\frac{1}{2} e^{i \xi}\left(1-e^{-i \xi}\right)^{2}, \\
& \widehat{\widetilde{a}}_{1}(\xi)=\frac{3}{8} \\
& \widehat{a}_{2}(\xi)=-\frac{1}{8}\left(4+e^{i \xi}+e^{-i \xi}\left(1-e^{-i \xi}\right)^{2},\right. \\
& \widehat{\tilde{a}}_{2}(\xi)=\frac{1}{8}\left(1+4 e^{-i \xi}+e^{-i 2 \xi}\right) .
\end{aligned}
$$

Then, this group of masks satisfy (20). Hence, the system pair $(X(\Psi), X(\widetilde{\Psi}))$ defined in (8) and (10) associated with the masks $\left\{\widehat{a}_{0}, \widehat{a}_{1}, \widehat{a}_{2}\right\},\left\{\widehat{\widetilde{a}}_{0}, \widehat{\widetilde{a}}_{1}, \widehat{\widetilde{a}}_{2}\right\}$ is a dual wavelet frame for $L_{2}(\mathscr{M})$.

Example 19 (pseudo). Let $\widehat{a}_{0}(\xi)=\cos ^{4}(\xi / 2)$ and $\widehat{\widetilde{a}}_{0}(\xi)=$ $\cos ^{4}(\xi / 2)\left(1+4 \sin ^{2}(\xi / 2)\right.$ be the mask of the m order pseudospline. Define

$$
\begin{aligned}
& \widehat{a}_{1}(\xi)=e^{-i \xi} \sin ^{4}\left(\frac{\xi}{2}\right)\left(1+4 \sin ^{2}\left(\frac{\xi}{2}\right)\right), \\
& \widehat{\widetilde{a}}_{1}(\xi)=e^{i \xi} \sin ^{4}\left(\frac{\xi}{2}\right), \\
& \widehat{a}_{2}(\xi)=\frac{\sqrt{5}}{4} \sin ^{2}(\xi), \\
& \widehat{\widetilde{a}}_{2}(\xi)=\frac{\sqrt{5}}{4} \sin ^{2}(\xi), \\
& \widehat{a}_{3}(\xi)=e^{-i \xi} \frac{\sqrt{5}}{4} \sin ^{2}(\xi), \\
& \widehat{\widetilde{a}}_{3}(\xi)=e^{i \xi} \frac{\sqrt{5}}{4} \sin ^{2}(\xi) .
\end{aligned}
$$

Then, this group of masks satisfy (20). Hence, the system pair $(X(\Psi), X(\widetilde{\Psi}))$ defined in (8) and (10) associated with the masks $\left\{\widehat{a}_{0}, \widehat{a}_{1}, \widehat{a}_{2}, \widehat{a}_{3}\right\},\left\{\widehat{\widetilde{a}}_{0}, \widehat{\widetilde{a}}_{1}, \widehat{\widetilde{a}}_{2}, \widehat{\widetilde{a}}_{3}\right\}$ is a dual wavelet frame for $L_{2}(\mathscr{M})$. 
TABLE 1: Approximation errors $\left\|\mathscr{T}_{j}^{n *}-\widehat{a}_{j}^{*}\right\|_{\infty}$ and $\left\|\widetilde{\mathscr{T}}_{j}^{n}-\widehat{\widetilde{a}}_{j}\right\|_{\infty}$ with $\widehat{a}_{j}$ and $\widehat{\widetilde{a}}_{j}$ in Example 17.

\begin{tabular}{lccccccc}
\hline Errors & $\widehat{a}_{0}^{*}$ & $\widehat{a}_{1}^{*}$ & $\widehat{a}_{2}^{*}$ & $\widehat{\widetilde{a}}_{0}$ & $\widehat{\widetilde{a}}_{1}$ & $\widehat{\widetilde{a}}_{2}$ & Average error \\
\hline $\mathrm{n}=7$ & $3.40 \mathrm{E}-05$ & $6.78 \mathrm{E}-05$ & $3.471 \mathrm{E}-03$ & $3.40 \mathrm{E}-05$ & $1.53 \mathrm{E}-05$ & $3.40 \mathrm{E}-05$ & $6.09 \mathrm{E}-04$ \\
$\mathrm{n}=8$ & $3.73 \mathrm{E}-07$ & $7.27 \mathrm{E}-07$ & $7.12 \mathrm{E}-04$ & $3.73 \mathrm{E}-07$ & $3.13 \mathrm{E}-06$ & $3.40 \mathrm{E}-06$ & $1.20 \mathrm{E}-04$ \\
$\mathrm{n}=9$ & $3.51 \mathrm{E}-06$ & $5.44 \mathrm{E}-07$ & $1.27 \mathrm{E}-04$ & $3.51 \mathrm{E}-06$ & $4.13 \mathrm{E}-06$ & $3.07 \mathrm{E}-07$ & $2.31 \mathrm{E}-05$ \\
$\mathrm{n}=10$ & $3.75 \mathrm{E}-06$ & $3.91 \mathrm{E}-07$ & $2.03 \mathrm{E}-05$ & $3.75 \mathrm{E}-06$ & $4.06 \mathrm{E}-06$ & $6.08 \mathrm{E}-08$ & $5.38 \mathrm{E}-06$ \\
$\mathrm{n}=11$ & $3.89 \mathrm{E}-06$ & $3.44 \mathrm{E}-07$ & $2.98 \mathrm{E}-06$ & $3.89 \mathrm{E}-06$ & $4.12 \mathrm{E}-06$ & $4.41 \mathrm{E}-08$ & $2.54 \mathrm{E}-06$ \\
\hline
\end{tabular}

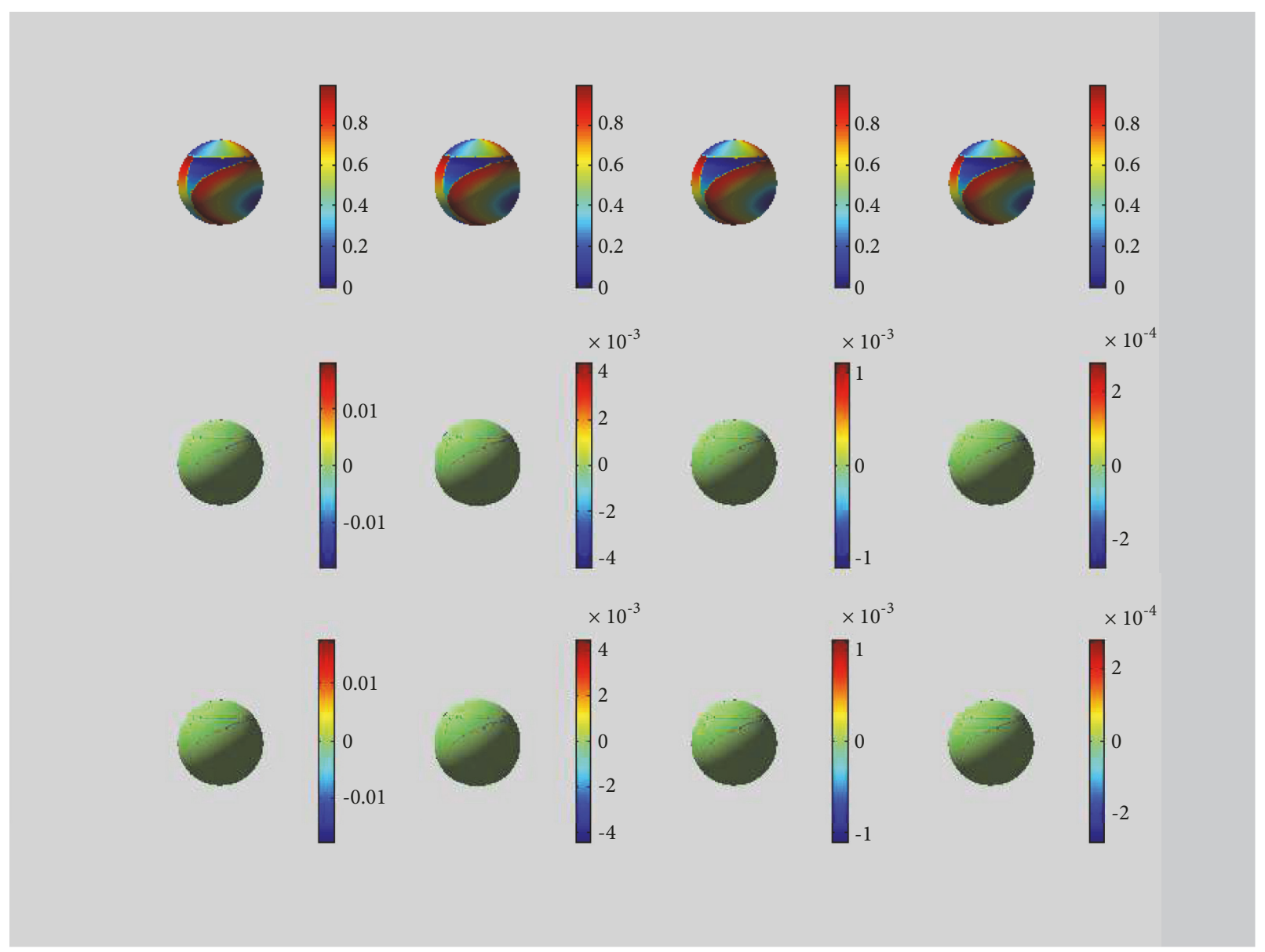

FIGURE 1: The figure shows that the graph data on Slope is decomposed to the dual wavelet frame coefficients $W_{j, l} f_{G}$ for $0 \leq j \leq 2$ (row 1-3) and $1 \leq l \leq 4$ (column $1-4$ ).

In our simulations, we choose the dual wavelet frame system given in Example 17. We accurately approximate the trigonometric polynomial masks $\widehat{a}_{j}^{*}$ and $\widehat{\tilde{a}}_{j}$ in Example 17 by low-degree Chebyshev polynomials; see Table 1 .

In numerical experiment, we take 16728 points sampled on the manifold and we build a weighted graph by setting edge weights $w\left(v_{m}, v_{n}\right)=e^{-\left\|v_{m}-v_{n}\right\|_{2}^{2} / \sigma}$ for $v_{m}, v_{n} \in V$. For larger datasets, this graph could be sparsified by thresholding the edge weights. We use $\sigma=10$ and threshold the adjacency matrix $A$ to limit the number of nearest neighbors of each vertex to 10 for computing the underlying weighted graph. The functions $f_{G}: V \longrightarrow \mathbb{R}$ are generated by mapping two images, Slope and Eric, onto the graph $G$; see Figure 2 of the literature [1]. We perform 4 levels of DWFTG and use $n=11$ for the Chebyshev polynomial approximation of the masks.

As described in Algorithm 13, we can decompose graph data on Slope and Eric to obtain the dual wavelet frame coefficients $W_{j, l} f_{G}$ for $0 \leq j \leq 2$ and $1 \leq l \leq 4$ by the decomposition of (63) (see Figures 1 and 2) and reconstruct graph data on Slope and Eric from these framelet coefficient sequences by the reconstruction of (66); see Figure 3 .

The graphs in Figures 1 and 2 show that the dual wavelet frame systems provide both good data approximation and detail separation based on multiresolution analysis; thus we demonstrate the ability of the fast dual wavelet frame transform for graph data analysis. 


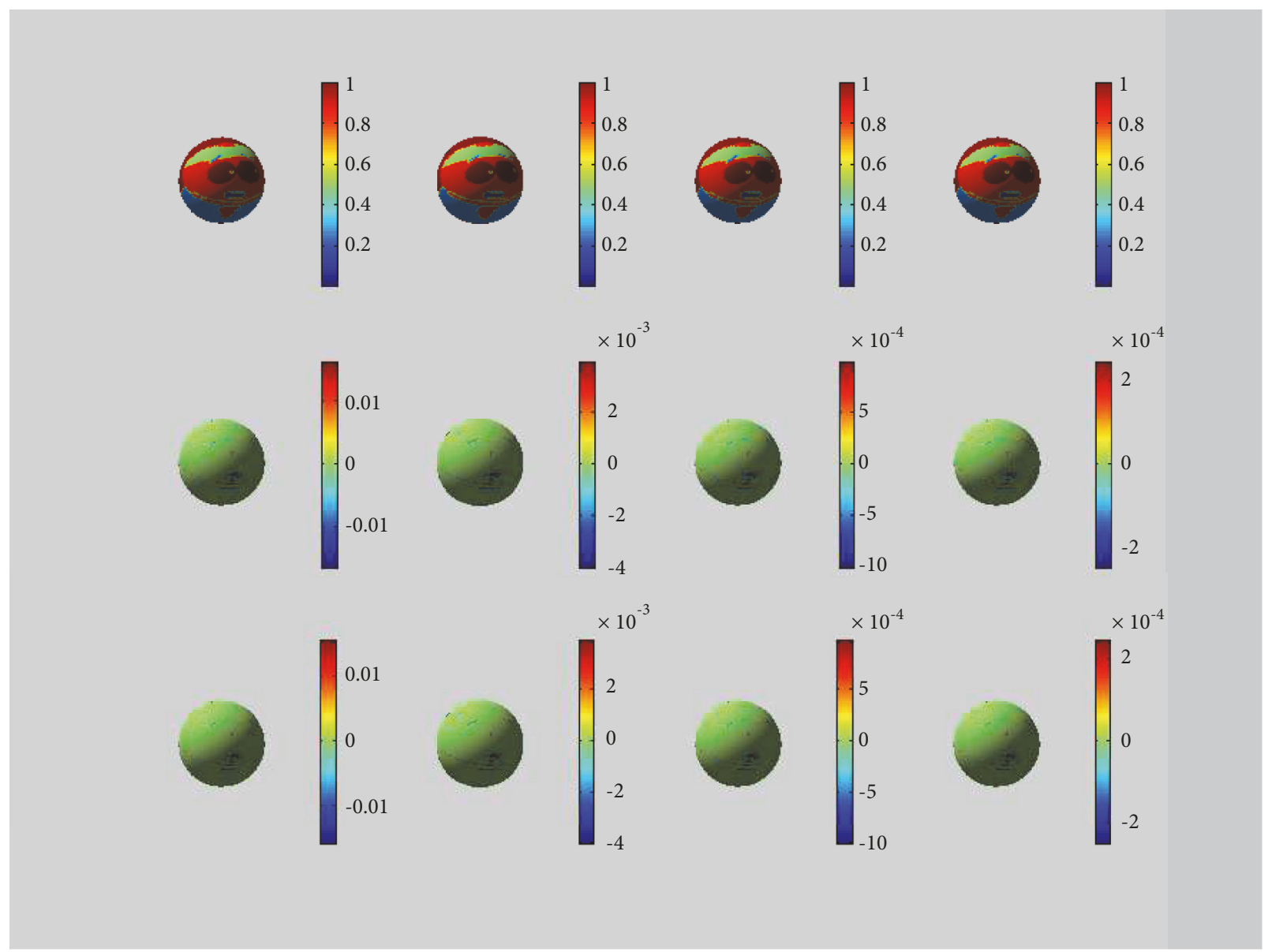

FIGURE 2: The figure shows that graph data on Eric is decomposed to the dual wavelet frame coefficients $W_{j, l} f_{G}$ for $0 \leq j \leq 2$ (row 1-3) and $1 \leq l \leq 4$ (column 1-4).
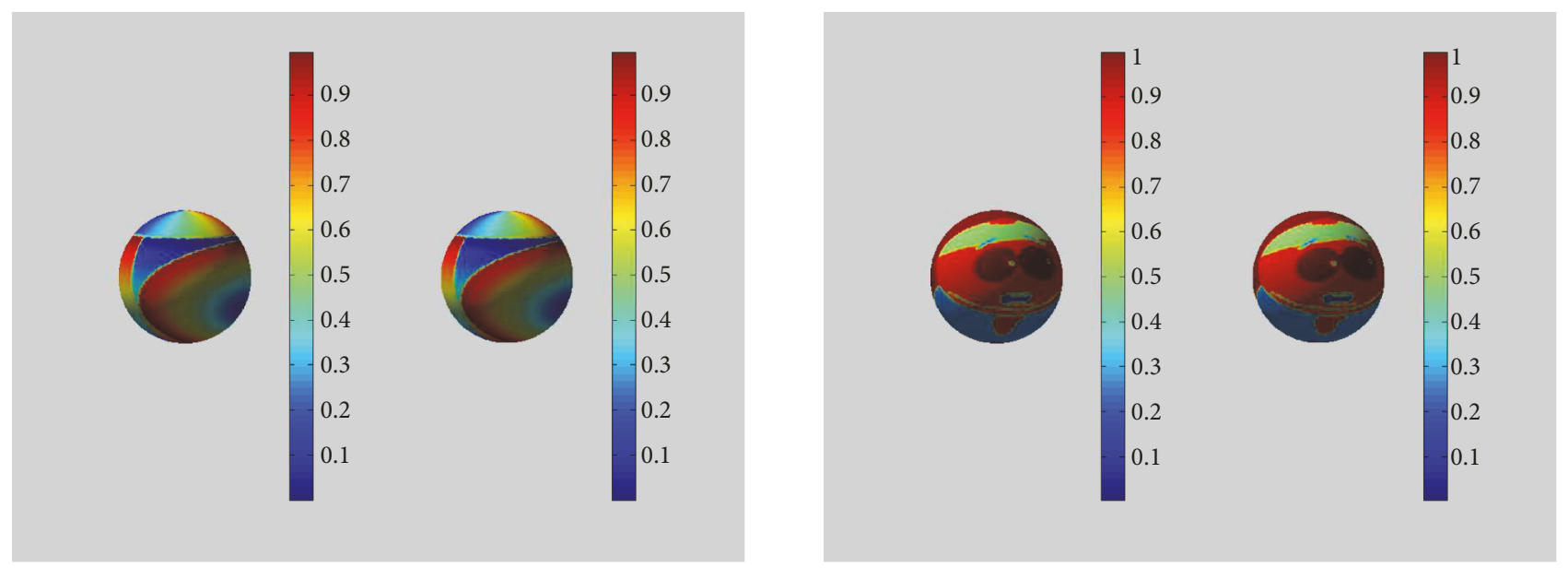

FIGURE 3: The graph data on Slope and Eric, respectively, reconstructed from corresponding framelet coefficient sequences are shown on the right of each image.

The computation time and reconstruction errors of a 4-level decomposition and reconstruction of the DWFTG are summarized in Table 2. For a similar computation time, the reconstruction error of DWFTG using linear masks is smaller than DWFTG using cubic masks and DWFTG using pseudo masks. However, the DWFTG using linear masks is a more redundant transformation than the DWFTG using cubic masks and DWFTG using pseudo masks. 
TABLE 2: The reconstruction time is in seconds and errors are measured by the $\ell_{\infty}$-norm.

\begin{tabular}{lcccccc}
\hline \multirow{2}{*}{$\begin{array}{l}\text { Image name } \\
\end{array}$} & Errors & Linear & \multicolumn{2}{c}{ Cubic } & \multicolumn{2}{c}{ Pseudo } \\
& Time(s) & Errors & Time(s) & Errors & Time(s) \\
\hline Slope & $2.570 \mathrm{E}-05$ & 5.960 & $9.526 \mathrm{E}-05$ & 5.912 & $5.686 \mathrm{E}-04$ \\
\hline Eric & $2.971 \mathrm{E}-05$ & 6.039 & $1.034 \mathrm{E}-04$ & 5.536 & $4.700 \mathrm{E}-04$ & 5.768 \\
\hline
\end{tabular}

Remark 20. Note that, the sparsity of the high frequency coefficients $W_{j, l} f_{G}$ is related to the support set of the scale function and the symmetry of the filter in image processing. When the support set of the scale function is smaller and the filter banks pair generating the dual wavelet frame system are symmetrical, the high frequency coefficients $W_{j, l} f_{G}$ are good sparse. On the contrary, the sparsity of the image is not guaranteed. At the same time, the reconstruction error will be slightly larger with the number of filters increasing. But the result is also ideal.

\section{Conclusion}

Wavelet frames on manifolds are a new research topic in the area of wavelet analysis. This paper is primarily concerned with the construction of the dual wavelet frames on manifolds and graphs using the machinery of extension principles. We have obtained the characterizations for the existence of dual wavelet frames in both the continuous and the discrete setting. In addition, we have also obtained the decomposition and reconstruction for the dual wavelet frame transforms on graphs. A complete section has been devoted to studying discrete dual wavelet frames on graphs using low-degree Chebyshev polynomials. Finally, some numerical simulations of fast dual wavelet frame transform on graphs have also been carried out to demonstrate the efficiency and accuracy of the framelet transforms.

\section{Data Availability}

The data used to support the findings of this study are available from the corresponding author upon request.

\section{Conflicts of Interest}

The authors declare that they have no conflicts of interest.

\section{Acknowledgments}

The authors appreciate the support of the National Natural Science Foundation of China (Grant no. 21676010). We would like to thank the authors and experts whose literatures are cited in this paper and those who have taken the time to review our paper.

\section{References}

[1] B. Dong, "Sparse representation on graphs by tight wavelet frames and applications," Applied and Computational Harmonic Analysis, vol. 42, no. 3, pp. 452-479, 2017.
[2] R. R. Coifman and M. Maggioni, "Diffusion wavelets," Applied and Computational Harmonic Analysis, vol. 21, no. 1, pp. 53-94, 2006.

[3] M. Maggioni and H. N. Mhaskar, "Diffusion polynomial frames on metric measure spaces," Applied and Computational Harmonic Analysis, vol. 24, no. 3, pp. 329-353, 2008.

[4] D. Geller and A. Mayeli, "Continuous wavelets on compact manifolds," Mathematische Zeitschrift, vol. 262, no. 4, pp. 895927, 2009.

[5] T. Hou and H. Qin, "Continuous and discrete Mexican hat wavelet transforms on manifolds," Graphical Models, vol. 74, no. 4, pp. 221-232, 2012.

[6] D. K. Hammond, P. Vandergheynst, and R. Gribonval, "Wavelets on graphs via spectral graph theory," Applied and Computational Harmonic Analysis, vol. 30, no. 2, pp. 129-150, 2011.

[7] N. Leonardi and D. Van De Ville, "Tight wavelet frames on multislice graphs," IEEE Transactions on Signal Processing, vol. 61, no. 13, pp. 3357-3367, 2013.

[8] N. Leonardi and D. Van De Ville, "Tight wavelet frames on multislice graphs," in Proceedings of the IEEE Transactions on Signal Processing, vol. 61, pp. 2136-2139, 2011.

[9] D. I. Shuman, C. Wiesmeyr, N. Holighaus, and P. Vandergheynst, "Spectrum-adapted tight graph wavelet and vertexfrequency frames," IEEE Transactions on Signal Processing, vol. 63, no. 16, pp. 4223-4235, 2015.

[10] Y. G. Wang and X. Zhuang, "Tight framelets and fast framelet transforms on manifolds," Applied and Computational Harmonic Analysis, pp. 1-29, 2016.

[11] S. K. Narang and A. Ortega, "Compact support biorthogonal wavelet filterbanks for arbitrary undirected graphs," IEEE Transactions on Signal Processing, vol. 61, no. 19, pp. 4673-4685, 2013.

[12] Y. Tanaka and A. Sakiyama, " $M$-channel oversampled graph filter banks," IEEE Transactions on Signal Processing, vol. 62, no. 14, pp. 3578-3590, 2014.

[13] B. Han, "On dual wavelet tight frames," Applied and Computational Harmonic Analysis, vol. 4, no. 4, pp. 380-413, 1997.

[14] C. K. Chui, W. He, and J. Stöckler, "Compactly supported tight and sibling frames with maximum vanishing moments," Applied and Computational Harmonic Analysis, vol. 13, no. 3, pp. 224262, 2002.

[15] I. Daubechies and B. Han, "Pairs of dual wavelet frames from any two refinable functions," Constructive Approximation, vol. 20, no. 3, pp. 325-352, 2004.

[16] M. Ehler, "On multivariate compactly supported bi-frames," Journal of Fourier Analysis and Applications, vol. 13, no. 5, pp. 511-532, 2007.

[17] B. Han and Z. Shen, "Dual wavelet frames and Riesz bases in Sobolev spaces," Constructive Approximation, vol. 29, no. 3, pp. 369-406, 2009.

[18] Q. Jiang and D. K. Pounds, "Highly symmetric bi-frames for triangle surface multiresolution processing," Applied and 
Computational Harmonic Analysis, vol. 31, no. 3, pp. 370-391, 2011.

[19] L. Borup, R. Gribonval, and M. Nielsen, "Bi-framelet systems with few vanishing moments characterize Besov spaces," Applied and Computational Harmonic Analysis, vol. 17, no. 1, pp. 3-28, 2004.

[20] B. Han, "Recent developments on dual wavelet frames," Representations, Wavelets, and Frames, pp. 103-130, 2008.

[21] B. Han, "Pairs of frequency-based nonhomogeneous dual wavelet frames in the distribution space," Applied and Computational Harmonic Analysis, vol. 29, no. 3, pp. 330-353, 2010.

[22] A. Ron and Z. Shen, "Affine systems in $L^{2}(\mathbb{R})^{d}$ II: dual systems," Journal of Fourier Analysis and Applications, vol. 3, no. 5, pp. 617-637, 1997.

[23] I. Chavel, Eigenvalues in Riemannian Geometry, Academic Press Inc., Orlando, Fla, USA, 1984.

[24] H. Weyl, "Das asymptotische Verteilungsgesetz der Eigenwerte linearer partieller Differentialgleichungen (mit einer Anwendung auf die Theorie der Hohlraumstrahlung)," Mathematische Annalen, vol. 71, no. 4, pp. 441-479, 1912.

[25] D. Grieser, "Uniform bounds for eigenfunctions of the Laplacian on manifolds with boundary," Communications in Partial Differential Equations, vol. 27, no. 7-8, pp. 1283-1299, 2002.

[26] M. Belkin and P. Niyogi, "Towards a theoretical foundation for Laplacian-based manifold methods," in Learning theory, vol. 74, pp. 486-500, Springer, Berlin, Germany, 2005.

[27] M. Hein, J.-Y. Audibert, and U. Luxburg, "From graphs to manifolds;- weak and strong pointwise consistency of graph Laplacians," in Proceedings of the Conference on Learning Theory, pp. 470-485, Springer.

[28] E. Gine and V. Koltchinskii, Empirical Graph Laplacian Approximation of Laplace-Beltrami Operators: Large Sample Results, Lecture Notes-Monograph Series 51, 2006. 


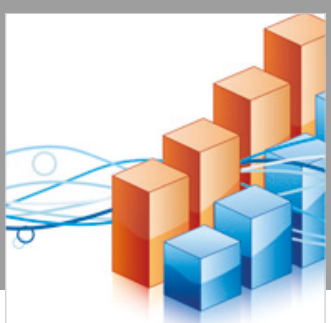

Advances in

Operations Research

\section{-n-m}
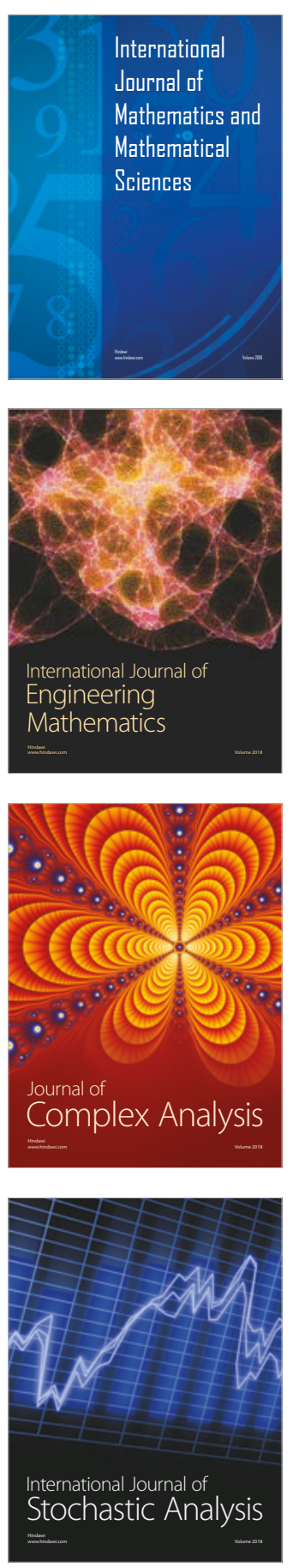
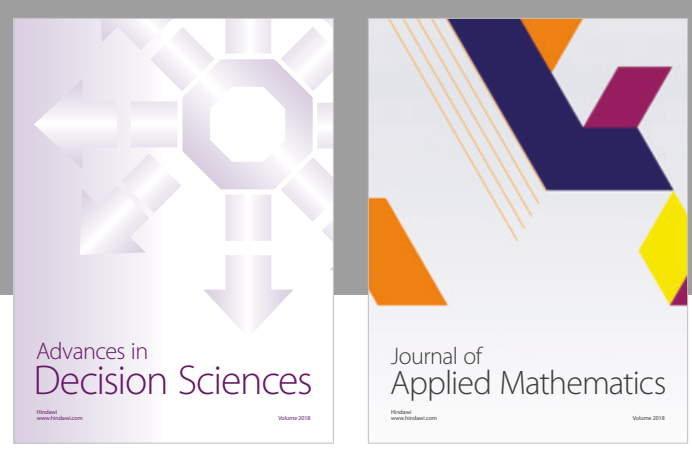

Journal of

Applied Mathematics
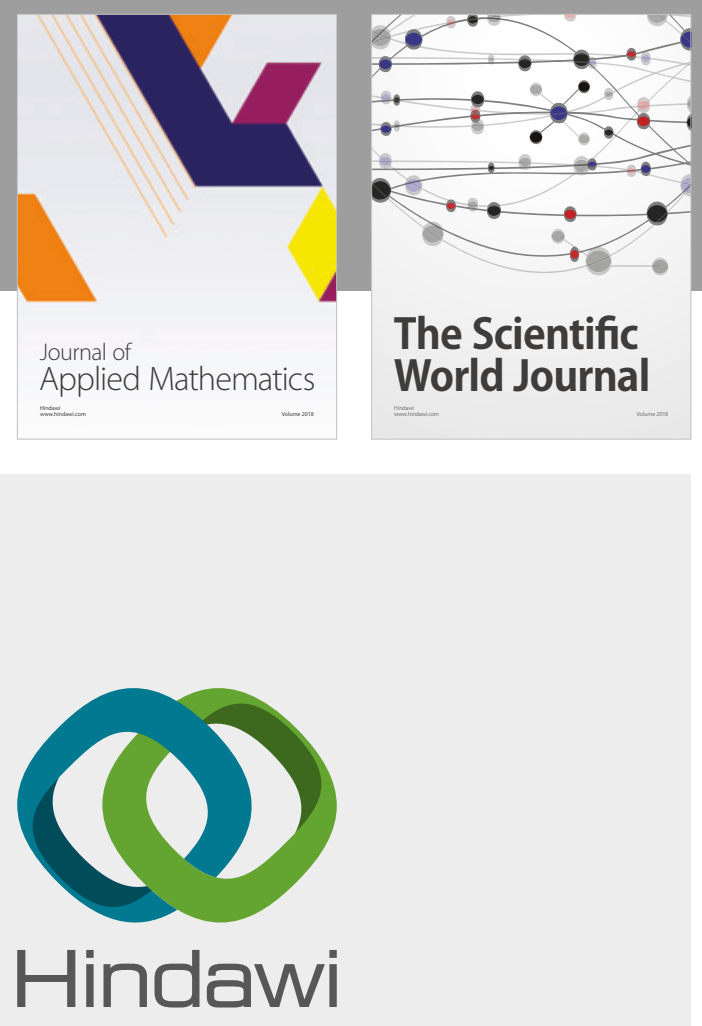

Submit your manuscripts at

www.hindawi.com

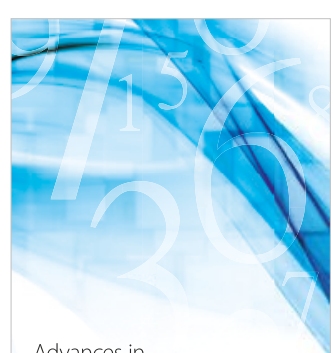

Advances in
Numerical Analysis
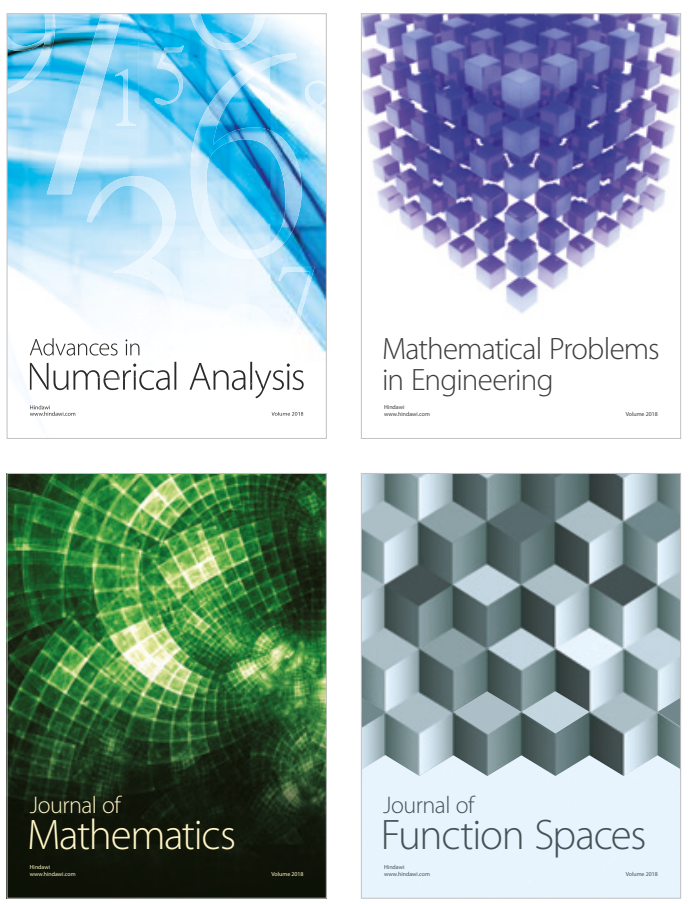

Mathematical Problems in Engineering

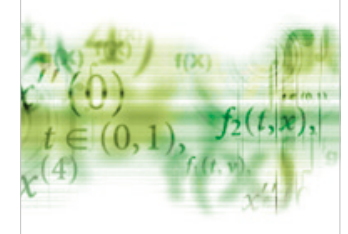

International Journal of

Differential Equations

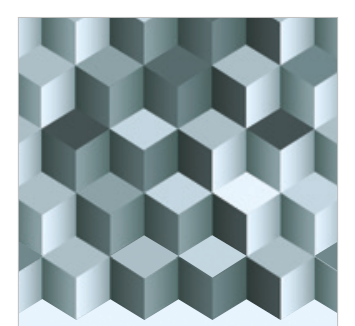

Journal of

Function Spaces

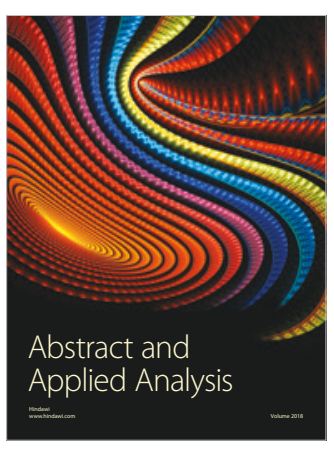

The Scientific

World Journal

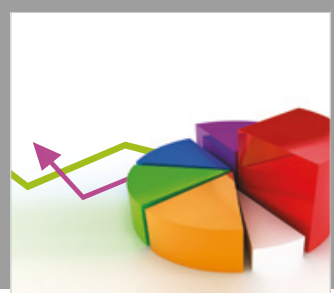

Journal of

Probability and Statistics
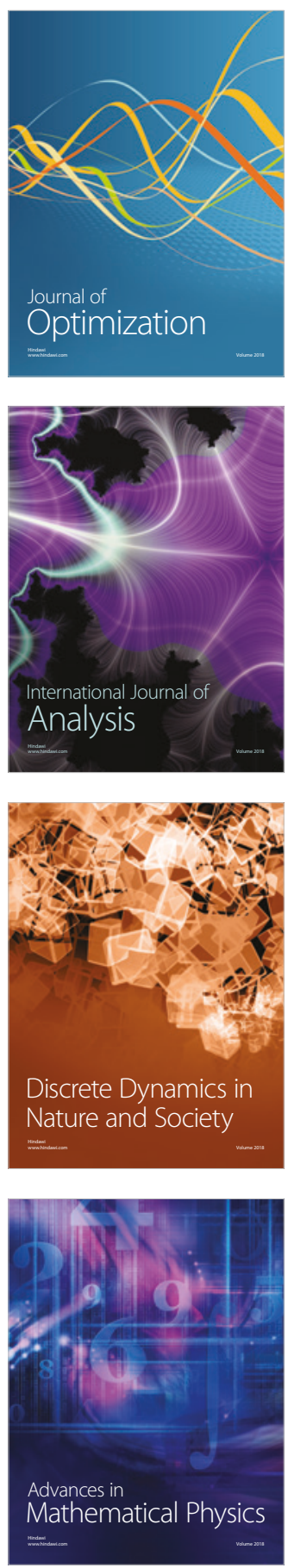